\title{
How to Apply TPM in Equipment Management for Chinese Enterprises
}

\author{
CHEN Li-xia, MENG Bo \\ Changchun University of Science and Technology, Changchun, China
}

\begin{abstract}
The paper studies an effective way for Chinese enterprises to implement Total Productive Maintenance (TPM) to cut cost and increase efficiency under the impact of financial crisis. It first reviews the definition and objectives of TPM, clarifies how TPM supports lean objectives, and suggests seven steps and essential elements for Chinese enterprises to implement TPM systematically and successfully. The paper also puts the steps and essential elements into practice in a Chinese enterprise, which are proven effective and we hope the proposals are helpful for Chinese enterprises to change their extensive operation in equipment management.
\end{abstract}

Keywords: TPM, autonomous maintenance, seven steps, essential elements

\section{Introduction to TPM}

Although TPM originated from preventive maintenance of USA in 1960s, it was put forward and got great success in 1981 in a Japanese company named Nippon Denso (Nakajima, 1989), which was spread worldwide from then on. With the impact of the financial crisis, many Chinese enterprises select to pull through by reducing wages, having long holiday, cutting budget, controlling traveling expenses and even canceling training plan to lower cost. From long-term view, the financial crisis also provides the best opportunity for Chinese enterprises to strengthen their foundation by implementing kaizen activities and TPM is just a tool for them to decrease cost and increase efficiency.

\section{The Definition of TPM}

TPM focuses primarily on manufacturing (although its benefits are applicable to virtually any "process”), and it is the first methodology Toyota used to improve its global position (Womack, 1990). We define TPM as a manufacturing initiative for optimizing the effectiveness of equipment by focusing on entire life of the machine, which involving all the contributors through active participation and teaming, making the operators the first line of defense against deterioration through autonomous maintenance, in other words, TPM is used to do everything possible to make sure that the machine could keep making good quality parts at the desired production rate every time you want it to.

\section{The Objectives of Implementing TPM}

The implementation of TPM will increase productivity within the total organization, where:

(1) Bring the equipment's condition to optimum level that ensures high level of availability;

CHEN Li-xia, Ph.D., School of Economics and Management, Changchun University of Science and Technology.

MENG Bo, bachelor, associate researcher, Industry Management Department, Changchun University of Science and Technology. 
(2) Develop a maintenance program for the entire life of the equipment that ensures continuous improvement;

(3) Develop the necessary standard procedures and checklists;

(4) Train the operators and maintenance personnel;

(5) Institutionalize good housekeeping through 5S;

(6) Involve personnel from all departments and all levels through support, participation and ownership;

(7) Implement and use an objective measurement system (OEE) to identify action items and monitor progress.

\section{How TPM Supports Lean Objectives}

Lean is a business environment where waste is identified continuously and eliminated passionately, TPM can add value and support lean objectives, just as Seiichi Nakajima (1989) said, “TPM can continuously improve all operational conditions within a production system by stimulating the daily awareness of all employees".

(1) Absence of breakdown maintenance:

Higher productivity of machine and operators;

Better utilization of maintenance staff.

(2) Reduced quality losses:

Machines produce quality parts all the time.

(3) Reduced consumables cost:

Reduced consumption of lubricants, coolants, etc.;

Reduced consumption of electricity, air, etc.;

Increased perishable tooling life.

(4) Longer productive life of machines and fixtures.

In addition to above-mentioned benefits of TPM, it also can reduce capital need and improve employed capital's utilization by improving equipment availability. For example, let us assume that through effective utilization of existing equipment, we reduce the capital need by $10 \%$, capital reduced by $\$ 300$ million, interest reduced by approximately $\$ 24$ million per year (interest rate 8\%) and net profit increased by 8.5\%.

\section{The Seven Steps to Implement TPM}

TPM is a long journey and systematic engineering, but too many studies are long on the theories but scanty on the implementation details. We put forward seven steps for Chinese enterprises to implement TPM step by step and get success in it as shown in Figure 1.

\section{Step 1-Perform Initial Cleaning and Inspection}

Objectives of step 1. The objectives of step 1 are to clean the entire machine inside and out, inspect for problems and abnormalities anywhere in the machine, identify opportunities for improvements/enhancements to the original design, identify the routine cleaning and inspection needs, let operators and maintenance personnel learn how to thoroughly clean and inspect while cleaning, how to identify problems and abnormalities, and how to develop standards for routine activities.

Kaizen activities during step 1. In order to realize the objectives of this step, the kaizen activities include but not limit to observe the machine in running condition, if there is vibration, heat, noise, leakage, coolant splattering, chips falling, take pictures to capture as-is condition, implement all the safety measures, clean dirt, 
grime, swarf, chips, other contaminations from the outside and look for abnormalities, disassemble subsystems and components, clean them and list abnormalities, opportunities for improvement, components that need replacement and ideas for cleaning, inspection and lubrication standards.

\section{Step 2-Inspect for Problems, Abnormalities Anywhere in the Machine}

Objectives of step 2. The objectives of step 2 are to prevent accelerated deterioration by stopping the contaminants at their source and prevent introduction of contamination, learn about equipment improvement activities, make thorough cleaning and inspection easier and less time-consuming, focus on obstacles to effective cleaning and inspection, encourage operators to use their creativity to control equipment and inspire proactive approach.

Kaizen activities during step 2. The kaizen activities during this step include but not limit to assign tasks, appoint a team leader, recorders, time-keeper, photographer and parts expeditor, assign specific tasks to all the other team members, observe the running machine, understand the machine's history, study the machine's operator and maintenance manuals, review maintenance history, analyze OEE data and causes of losses, review the quality and SPC data of the machine, take pictures to capture as-is condition, prepare the machine and the surrounding area, implement all the safety measures, build walls and dikes (if necessary), gather cleaning supplies and tools, clean contaminations from the outside and look for abnormalities, disassemble subsystems and components, clean them and list abnormalities, make sure parts of the disassembled machine do not get used for other similar machines, use guideline check sheets to ensure all items are covered, document abnormalities, areas to be improved and hard to access.

\section{Step 3-Develop Cleaning, Lubricating, and Inspection Standards}

Objectives of step 3. The objectives of step 3 are to capture the knowledge and understanding acquired by the operators and maintenance technicians, develop provisional standards for cleaning, inspection and lubrication, organize the work area and machine and place, realize visual controls to help follow standards efficiently in order to maintain equipment's condition achieved in step 1 and step 2.

Kaizen activities during step 3. During this step, TPM team must develop standards with very active participation of the operators and non-participating operators of the machine must review provisional standards before implementation, list the tasks in a logical, efficient sequence, verify appropriateness and safety of the tasks, audit operator capability and skills.

\section{Step 4-Provide Basic Training in Machine's Function and Controls to Enable Effective General Inspection}

Objectives of step 4. The objectives of step 4 are to enhance operator's knowledge and skills related to equipment's operation, systems and components, improve inspection and diagnostic skills, expand the scope of routine inspection performed by the operators, promote continuous improvement through corrective and predictive maintenance, and make sure there is a common "language" and terminology throughout the organization.

Kaizen activities during step 4. The kaizen activities during this step include: identify training topics, develop training material and methods, evaluate the potential trainer resources and select, develop a master plan for training delivery, provide the training, evaluate the training performance and make necessary changes and periodically reevaluate the training needs.

\section{Step 5-Enhance and Implement Autonomous Inspection Standards and Schedules}

Objectives of step 5 . The objectives of step 5 are to update and finalize the provisional standards developed 
during step 3, update and finalize schedules for autonomous maintenance activities, preventive maintenance and audits, develop and institutionalize improvement process for the standards, combine cleaning, lubrication and inspection tasks into "unified autonomous maintenance standards" in order to substantially reduce the time taken for cleaning, lubrication and inspection activities.

Kaizen activities during step 5. The kaizen activities during this step include: verify the ability and understanding of each operator, perform trial runs of standard activities for each operator, ensure adherence to the standards and sequence by all the operators, time each operator and make the necessary improvements through further training, complete all the pending workplace organization activities from step 3, verify effectiveness of the visual elements supporting TPM, start developing the audit standards and details, place up-to-date standards in all the TPM manuals.

\section{Step 6-Implement Workplace Organization and Housekeeping by Employing $5 S$ and Visual Controls}

Objectives of step 6. The objectives of step 6 are to maintain the area surrounding the machine clean and organized at all times to support the disciplined TPM activities, implement and sustain $5 \mathrm{~S}$ to establish an environment that is safe, a surrounding helps efficient working, "Visual workplace” helps avoid mistakes and helps train new employees easily, so that to promote a sense of ownership and pride in the workplace.

Kaizen activities during step 6. The kaizen activities during this step mainly include two parts, one is $5 \mathrm{~S}$ and the other is to make workplace visual.

(1) Employ 5S

$5 \mathrm{~S}$ that is the basis for lean manufacturing and the foundation for a disciplined approach to the workplace including five steps (Chen \& Meng, 2008):

(a) Sort involves sorting through the contents of the workplace and removing unnecessary items.

(b) Straighten involves putting the necessary items in their place and providing easy access.

(c) Shine involves cleaning everything, keeping it clean daily and using cleaning to inspect the workplace and equipment for defects.

(d) Standardize involves creating visual controls and guidelines for keeping the workplace organized, orderly, and clean.

(e) Sustain involves training and discipline to ensure that everyone follows the 5S standards.

(2) Make workplace visual

Visual workplace means all the necessary information is proactively displayed that helps avoid mistakes and provides status at a glance (Leflar, 2001). Usually, following items in workplace should be made visual for TPM:

- TPM Team's story board;

- All the info on the project, plans, achieved results, etc.;

- TPM Standards, schedules, assignments and real-time status update;

- Instructions for safe operations and warnings;

- Instructions for inspection and lubrication activities;

- Location markings of every needed item in the area;

- Markings to highlight normal and abnormal conditions;

- Special instructions for unscheduled actions.

Step 7-Follow up on All the Above Steps and Advanced Improvement Activities

Objectives of step 7. The objectives of step 7 are to put in place mechanisms that will ensure continuing 
deployment and sustenance of autonomous maintenance, develop and deploy an effective audit process, implement beneficial results to other similar machines.

Kaizen activities during step 7. The activities during is to continuously evaluate how far you are from the goal of zero breakdown and attempt to reduce it by any activities possible.

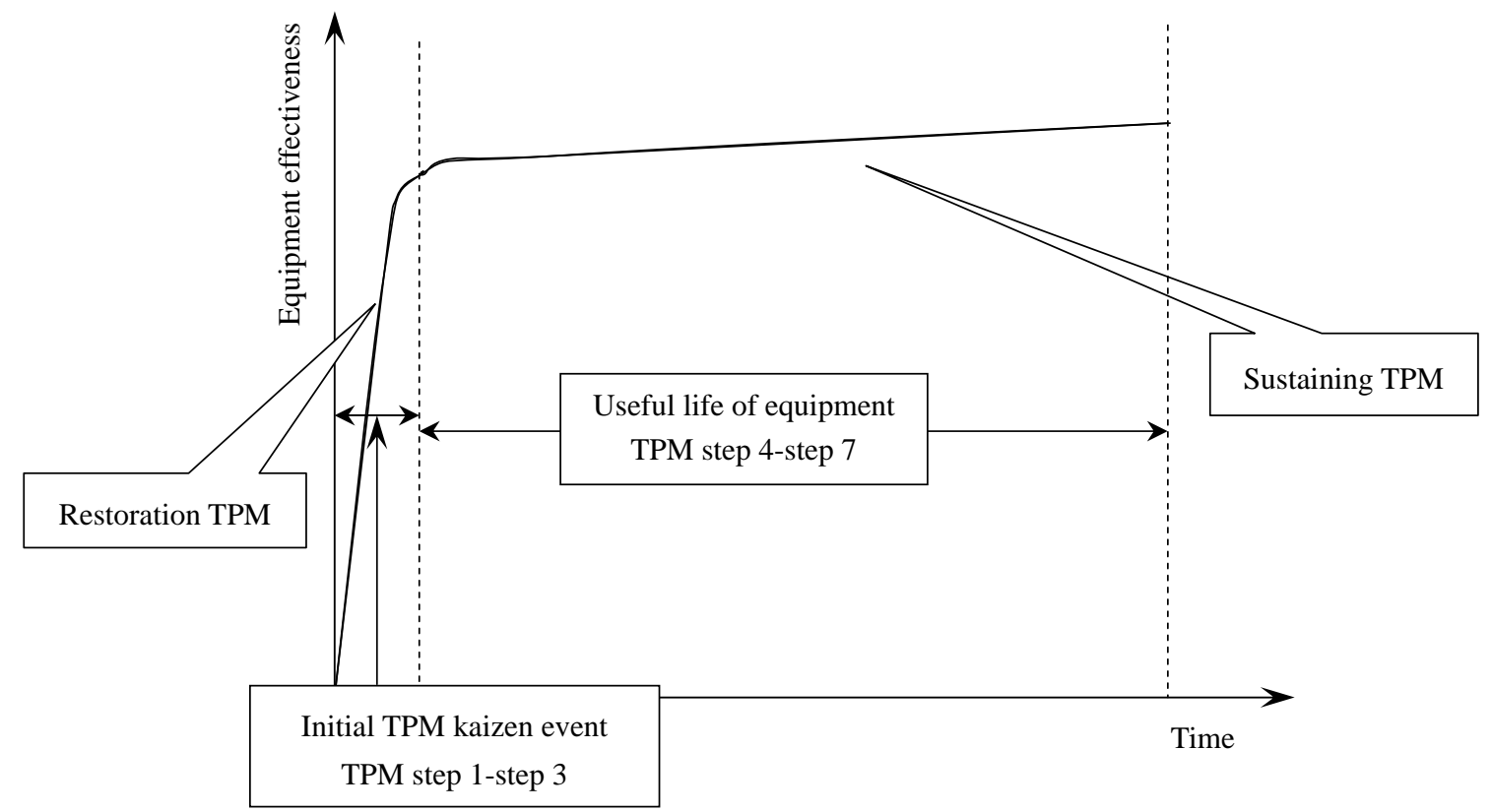

Figure 1. Seven steps to implement TPM.

\section{Essential Elements for Successful TPM Program}

In so many Chinese enterprises, first-line operators are only responsible for operate equipments and careless of their basic maintenance due to lack of autonomous maintenance concept or skills, maintenance employees simply replace non operative components and take it as a maintenance that is a temporary solution, and professional engineers are busy in coping with emergency, having no time and energy in implementing equipment management and not mention to equipment improvement. So, we propose following essential elements for Chinese enterprises to implement TPM successfully.

\section{Well-Done Preparation for TPM}

Sufficient preparation is half of a success, enterprises must prepare adequately in advance for implementing TPM smoothly and successfully, which at least includes following preparations.

Establish a TPM team. TPM requires more time, resources and efforts than most of Chinese enterprises believe they can afford. A typical TPM implementation requires company-wide participation and full results can only be seen after 3 years and sometimes 5 years. It is proven that an efficient TPM team can reduce this process greatly.

(1) The members of TPM team

Ideal number of team members is 4-7 and it should include all the operators of the machine (all shifts), the maintenance technicians specialized in mechanical, hydraulics, electronics, etc., manufacturing engineer, departmental supervisor, one or two "outside" operators and one member of the management.

(2) The leader of TPM team

Team leader should be closely associated with the machine, preferably the machine operator or maintenance 
technician, who must believe in discipline, accountability and open communication, and is able to "coach" the team into taking ownership of the machine, provide team training prior to TPM event and motivate the team to make TPM more smooth and successful.

A carefully designed TPM plan. Planning must start two months before TPM event, in which:

- Select the candidate machine that is critical on the value stream mapping, make sure there is a backup machine with sufficient capacity, available to take up this machine's load and generate sufficient inventory of parts.

- Gather all the available information for this machine, for example, the user manuals, maintenance manuals, control circuit diagrams, drawings, the maintenance history for at least two years and the OEE data for this machine.

- Identify repeating and chronic problems by discuss with the operators and maintenance technician.

- Develop an action plan for the machine during TPM event including identifying a secured location to store all the new and disassembled and cleaned spares for the machine, developing a plan for disassembling all the major machine components, and a plan for washing and painting the machine components, and the base that may not be moved, ordering sufficient quantities of various fasteners, bearings, seals, plugs, gages, v-belts, chains, lubricants, coolants, etc. that are certain to be replaced, making a list of all the miscellaneous spares, supplies, hardware, etc. that may be needed, make sure all the necessary hand tools and power tools are available in sufficient quantity in order to quickly bring the machine in sunning condition.

\section{Active Participation of Everyone}

A successful TPM can't realize without active participation of everyone, besides the operators and maintenance technicians, the participation of the management and other departments are very important for TPM activities.

The involve of the management. The management in different level should provide complete, real and visible support for TPM, which is necessary for sustaining TPM program. Chinese people like to follow their leaders, so top management should actively participate in TPM event, provide resources and empower the TPM team, middle managers should participate in audits and TPM kaizen events, plant manager should give the awards with fanfare to encourage the morale.

Active participation of all staff. All departments' involvement is critical for an enterprise to successfully implement TPM. TPM is not a single task, besides the operators and maintenance technicians who work directly on the machine; it also needs the active participation of the staff of manufacturing engineering, maintenance, production, IT, etc..

\section{A Culture of Autonomous Maintenance}

Absolute faith and commitment of operators and maintenance technicians are the basis of a successful TPM. Because they spend the most time with the machines, so they can and will notice the abnormalities first, have the most unique knowledge of the machine, it's parts and it's functioning, have much closer emotional attachment with, and sense of ownership of the machines and are mostly technically inclined, so they are the "first line of defense” for the machine. We should provide them with adequate training and empowerment to ensure regular cleaning, lubrication, inspections and total ownership that achieves continuous improvement in equipment effectiveness, and more important, we should change their attitude from passive to autonomous maintenance since it is self-governing, self-ruling, non-centralized machine maintenance program. 


\section{Case Study: The Application of TPM in Jilin Huawei Company}

Jilin Huawei Company (Huawei hereafter) is a large-scale plant that supply parts and components for FAWVolkswagen. Huawei conducted TPM in March 2006, supported by a professional management consulting company for three months, during which the authors were guiding our students of production practice in the production site. Although it got instant effect, it restored to its original state soon after the consulting experts left and many employees including the management and first-line operators thought TPM as a form of cleaning movement without long-term effect, which made the general manager felt embarrassed not only because of the consulting fee he paid for the consulting company, but also the employees' suspicion on TPM that is proven a effective way in the world. We decided to help him find out a way of implementing TPM with long-term effect and without cost increase since he is worthy of our esteem in respect that he has provided practice opportunity for our students, and more important, we are extremely familiar with the status of the factory. We conduct TPM as the steps above-mentioned and pay attention to the essential elements from the beginning of 2007.

\section{Establish a TPM Team}

There are seven members in the TPM team including the GM who promise that he will earnestly practice TPM by providing the resources needed, three operators of machine A, the workshop director, a maintenance technician and a manufacturing engineer, and the leader of the team is the workshop director.

\section{TPM Team Design TPM Plan Carefully and Train Employees}

We first train the TPM team and guide them to design a detailed TPM plan and train plan in order to make good preparation for deploying TPM. We especially emphasize the necessity and importance all staff's involvement and let them know that TPM is not a onetime event but a lifetime commitment, and it can't succeed without the culture of autonomous maintenance.

\section{Organize All Staff to Analyze the Problems of Huawei in Equipment Management}

We find that many of the problems in equipment management around the world are all universal. The difference is that some companies are recognizing these problems, adopting TPM and utilizing their work force in teams to solve the problems and others are not. So we design a Pre-TPM Checklist to help them analyze the problems of Huawei as shown in Table 1.

Due to our good preparation in advance, we got active participation of all staff in brainstorming the problems

\section{Deploy TPM Step by Step}

We implemented TPM in three stages according to the seven steps we propose as shown in Table 2 from 2007 to 2009.

The staff in Huawei put forward so many improvement ideas and most of them were realized as shown in Figure 2, therefore the overall situation of equipment management and employees' morale has changed greatly.

\section{Evaluate the Effect of TPM by OEE Index}

We use Overall Equipment Effectiveness (OEE) as the evaluation index of TPM that represents the percentage of time a machine was actually producing quality parts compared to the time it was planned to be. As shown in Figure 3, we improve the OEE of machine A from 35\% in 2007 to $70 \%$ in 2009 and we hope it will be $85 \%$ be the end of 2010 , which is a world class objective. 
Table 1

\section{Pre-TPM Check List}

\begin{tabular}{|c|c|}
\hline Cleanliness & $\begin{array}{l}\text { The equipment is generally dirty; } \\
\text { Equipment surfaces are covered with a cake of dirt and grime; } \\
\text { Metal chips / debris is scattered / accumulated on and around the equipment; } \\
\text { The equipment leaks hydraulic fluid; } \\
\text { Coolant splashes around outside the splash guards; } \\
\text { Motors are covered with oil, they get hot, and generate oil fumes or make unusual noises; } \\
\text { Limit switches are covered with chips and oil; } \\
\text { Equipment parts under the covers are not cleaned; } \\
\text { Fixtures, tools, gages, etc. are not always cleaned; } \\
\text { Tops of the work-benches and other working surfaces are not clean; } \\
\text { Floor space around the equipment is generally covered with dirt, chips, oil, coolant, etc.; } \\
\text { Several unneeded items are laying around on various parts of the equipment. }\end{array}$ \\
\hline & $\begin{array}{l}\text { Operators do not fully understand the equipment’s capabilities and limitations; } \\
\text { Operators are not responsible for keeping the equipment clean and in working order; } \\
\text { Equipment breaks down frequently without any indications; } \\
\text { Fixing the equipment takes a long time; } \\
\text { Equipment does not produce quality parts in a consistent manner; } \\
\text { Certain setups take up a lot of time due to the adjustments required on the equipment; } \\
\text { Overall Equipment Effectiveness (OEE) of the equipment is not tracked; } \\
\text { There are no designated places for hand tools, gages, oil cans, etc.; } \\
\text { There are no legible labels describing the functions of switches, push buttons, level indicators, gages, instruments, } \\
\text { warning lights, etc.. }\end{array}$ \\
\hline Maintenance & $\begin{array}{l}\text { There is no way to find out when certain parts of the equipment break; } \\
\text { Equipment maintenance is responsibility of maintenance department personnel only; } \\
\text { Some equipment parts are loose, they rattle and/or vibrate; } \\
\text { Walls, structures, other machines around the equipment make it difficult to perform maintenance checks; } \\
\text { Coolant drains are sometimes clogged up; } \\
\text { Cables and pipes are laid out in haphazard manner, making it difficult to figure out where they are coming from and } \\
\text { where they are going; } \\
\text { Several equipment parts are held with bail wires, strings, masking tape, etc.; } \\
\text { Lubricating all the necessary points of the equipment is not a regularly scheduled and assigned activity; } \\
\text { Operators do not know how many equipment locations require lubrication, or what type of lubrication is required; } \\
\text { Broken or defective instruments are not repaired or replaced. }\end{array}$ \\
\hline
\end{tabular}

Table 2

The Three Stage and Seven Steps in Deploying TPM

\begin{tabular}{|l|l|l|}
\hline \multicolumn{1}{|c|}{ Stage } & \multicolumn{1}{|c|}{ Steps } & \multicolumn{1}{|c|}{ Objective } \\
\hline Stage 1 & step 1 and step 2 & Restore the machine \\
\hline Stage 2 & step 3, step 4 and step 5 & Prepare for sustaining \\
\hline Stage 3 & step 6 and step 7 & Sustain \\
\hline
\end{tabular}

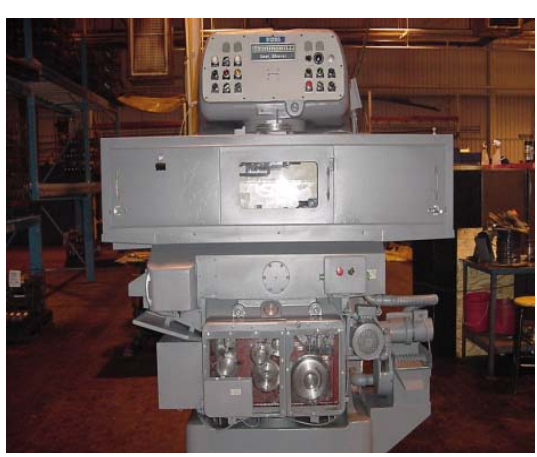

Transparent doors on the drives section

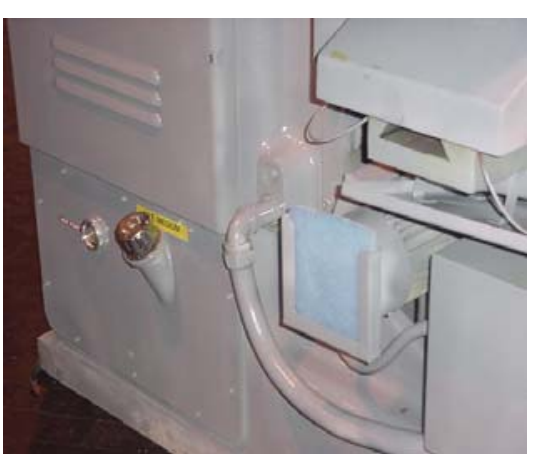

Transparent guard, chain number, acceptable range

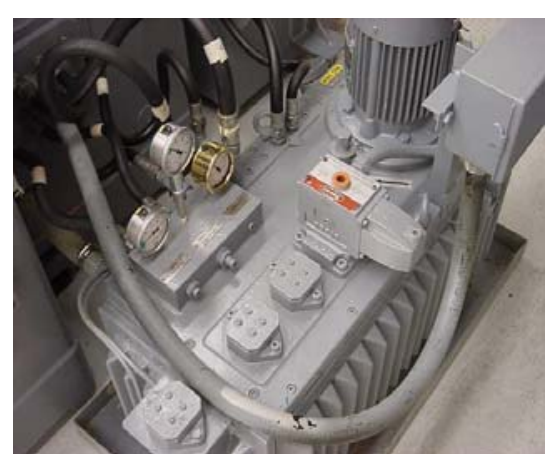

Visual indication for lubrication filling point and inspection point

Figure 2. Improvement ideas put forward and realized during deploying TPM. 


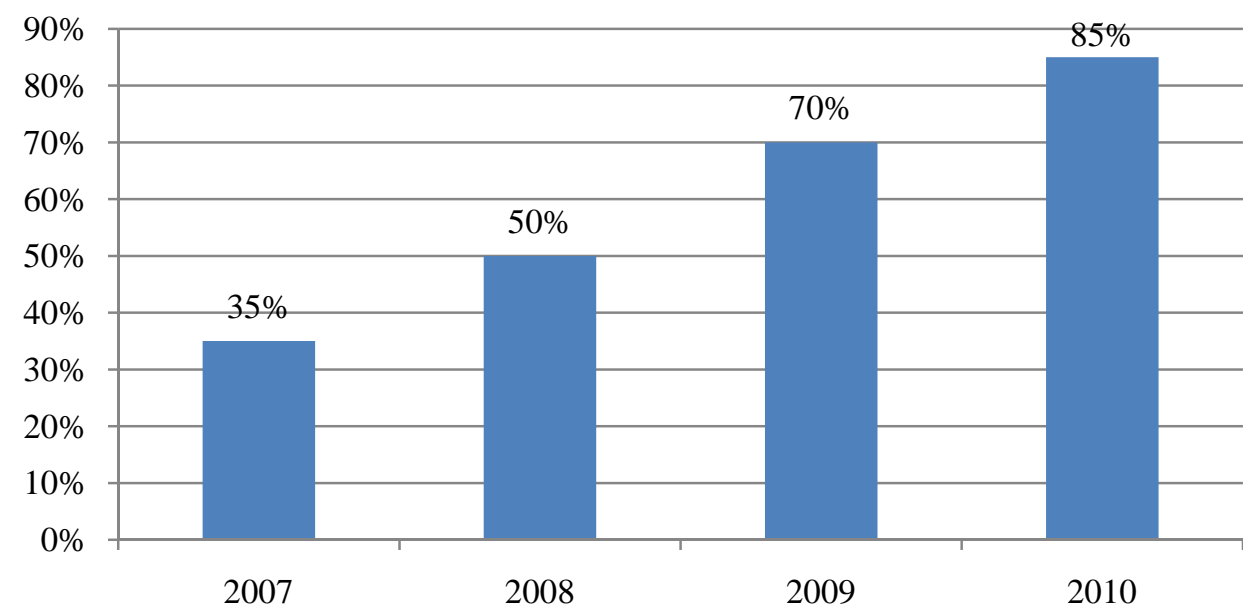

Figure 3. The OEE of machine A from 2007 to 2010.

\section{Conclusion}

TPM is a very useful lean tool for enterprises to eliminate wastes and improve production efficiency. The paper proposes a seven-step method for Chinese enterprises to implement TPM and especially suggests essential elements for their success in TPM, which are proven effective in Huawei. We herein thank Huawei a lot for giving us an opportunity to practice our research and we hope our study will benefit more Chinese enterprises.

\section{References}

CHEN, L. X., \& MENG, B. (2008). How to make 5S as a culture in Chinese enterprises. In Proceeding of 2008 International Conference on Information Management, Innovation Management and Industrial Engineering.

Leflar, J. A. (2001). Practical TPM: Successful equipment management at Agilent Technologies. Portland, Oregon: Productivity Press.

Nakajima, S. (1989). Introduction to TPM. Cambridge, Mass.: Productivity Press.

Womack, J. P., Jones, D. T., \& Roos, D. (1990). The machine that changed the world. New York: Macmillan Publishing Company. 